\title{
Genetic and Immunological Factors Involved in Natural Resistance to HIV-1 Infection
}

\author{
Natalia Taborda-Vanegas, Wildeman Zapata and María Teresa Rugeles*
}

\author{
Group of Immunovirology, University of Antioquia, Calle 62 \# 52-59, Medellin, Colombia
}

\begin{abstract}
Infection with Human immunodeficiency virus type-1 (HIV-1) induces severe alterations of the immune system leading to an increased susceptibility to opportunistic infections and malignancies. However, exposure to the virus does not always results in infection. Indeed, there exist individuals who have been repeatedly exposed to HIV-1 but do not exhibit clinical or serological evidence of infection, known as exposed seronegative individuals. Many studies have focused on the different mechanisms involved in natural resistance to HIV-1 infection, and have reported several factors associated with this phenomenon, including the presence of genetic polymorphisms in the viral coreceptors, innate and adaptive immune cells with particular phenotypic and functional features, and molecules such as antibodies and soluble factors that play an important role in defense against infection by HIV-1. The study of these factors could be the key for controlling this viral infection. This review summarizes the main mechanisms involved in resistance to HIV-1 infection.
\end{abstract}

Keywords: HIV-1 (Human immunodeficiency virus type-1), natural resistance, exposed seronegative individuals, polymorphisms, antibodies, soluble factors.

\section{INTRODUCTION}

Human immunodeficiency virus type-1 (HIV-1) infection remains one of the main public health problems in the world for which a vaccine is not yet available. In 2008 around 33.4 million persons were infected with HIV-1 throughout the world, with an annual incidence of 2.7 million infections, of which approximately $50 \%$ correspond to infections in women [1].

The natural history and development of HIV-1 infection is heterogeneous, if one considers the variability in the acquired immunodeficiency syndrome (AIDS)-free period exhibited by infected individuals; the median time of appearance of AIDS clinical symptoms is between 8 and 10 years after infection. However, cohort studies have identified a small fraction of seropositive (SPs) individuals, about 5$10 \%$, who despite infection for more than 10 years, remain symptomless and maintain a relatively high CD4+ cell count $(>500 \mathrm{CD} 4+$ cells $/ \mu 1)$ without antiviral therapy; they are known as long-term non-progressors (LTNPs) [2]. In addition, there are two new phenotypes of SP individuals who exhibit a spontaneous and sustained control of viral replication at least for one year in the absence of antiretroviral therapy; the first group is known as elite controllers since they maintain undetectable HIV RNA levels $(<50$ copies $/ \mathrm{mL}$ ), and the second group known as viremic controllers maintains detectable viral loads although $<2000$ copies/mL [3]. Likewise, individuals have been reported, who are frequently exposed to the virus by sexual, parenteral or vertical routes, but do not exhibit serological evidence of infection, or any signs or symptoms of immunodeficiency; they are known as high-risk exposed

*Address correspondence to this author at the Group of Immunovirology, University of Antioquia, Calle 62 \# 52-59, Lab 532, Medellín, Colombia; Tel: 57-4-2196482; Fax; 57-4-2196482;

E-mail:mtrugel@catios.udea.edu.co
HIV-seronegative or exposed uninfected (ESNs) individuals [2]. These groups have revealed the existence of natural resistance mechanisms against HIV. Several studies have been aimed at determining the various mechanisms involved in natural resistance to HIV infection, and so far it has been associated with immunological factors, host genetic variants as well as soluble factors that prevent or limit viral infection (Fig. (1) and Table 1). Among the genetic factors, the CCR5delta 32 (CCR5- $\Delta 32$ ) mutation has been the most widely investigated and is associated with natural resistance to HIV infection, because it efficiently inhibits entry of R5 strains; some treatments against HIV infection have even been developed based on these findings. In addition, other single nucleotide polymorphisms (SNPs) in HIV coreceptors and their natural ligands are involved in this process [4].

Several investigators have demonstrated the presence of immunological mechanisms that can reduce the risk of acquiring HIV infection or limit its progression. Certain reports indicate that apoptosis of target cells [5], increased production of interferon gamma (IFN- $\gamma$ ) by natural killer (NK) cells [6] and positive regulation of costimulatory molecules [7] are factors associated with resistance. The humoral response contributes significantly to resistance to HIV infection through the production of anti- HIV IgA in mucosal [8] and finally, several soluble factors such as RNases, chemokines, cytokines and cationic proteins are involved in blocking HIV infection in ESNs or in decreasing the time required to disease progression in LTNPs individuals [9].

In this review, we describe the main factors involved in natural resistance to $\mathrm{HIV}-1$ infection associated with ESN individuals, including some findings of our research group.

\section{ESNS GENETIC CHARACTERISTICS}

The differences in the susceptibility to HIV-1 infection are due partly to genetic characteristics, and many studies are 


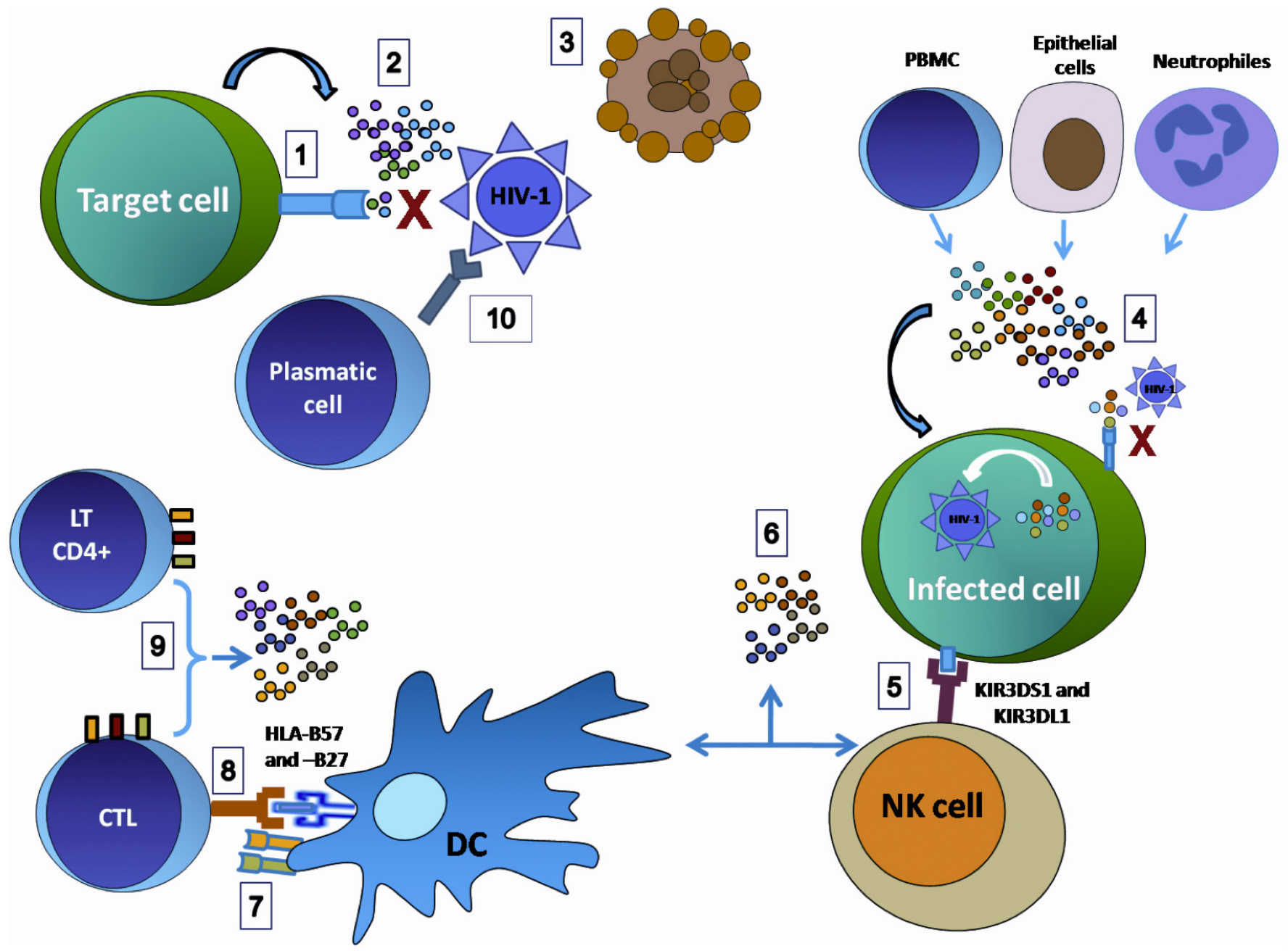

Fig. (1). Mechanisms of resistance involved in the protection against HIV-1-infection and AIDS-progression. Target cells have polymorphisms in CCR5 gene or other alternative viral coreceptors, such as CCR2 (1), all of them induce low or non-expression of these coreceptors, inhibiting viral entry. This step can be also blocked by high production of the coreceptors ligands, MIP-1 $\alpha / \beta$, RANTES and SDF-1 (2). Elevated spontaneous and inducible apoptosis of monocytes and other HIV-1-target cells has been reported in ESNs (3). Increased release of soluble antiviral factors APOBEC3G, CAF, $\alpha$ and $\beta$ - defensins, type I IFN, LIF, MIP- $1 \alpha / \beta$, RANTES, RNases, SDF-1, SLPI, TRIM5 $\alpha$ and elafin block viral entry or replication (4). ESNs individuals exhibit high activity in NK cells and DCs. NK cells express KIR3DS1 and KIR3DL1 related to cell activation (ㅁ) and are able to produce IFN- $\gamma(\underline{\mathbf{6}})$. DCs also express the co-stimulatory molecules CD80 and CD86 () $)$ and produce IFN- $\alpha(\underline{6})$. Type I IFN induces HLA expression, apoptosis of infected cells, production of antiviral molecules, and block viral replication. The specific immune response is characterized by the presence of CTLs restricted to HLA-B57 and B27, presenting immunodominant peptides (ㅇ), production of cytokines such as RANTES, MIP-1 $\beta$, IL-6, IFN- $\gamma$, TNF- $\alpha$ and $\beta$ and IL-2 that increase the immune response and expression of markers associated with T cell activation (9). B-cells produce neutralizing IgA antibodies, detectable in serum, mucosa and breast milk (10) .

aimed at identifying and establishing the relationship between the presentation of different polymorphisms and the lack of infection or delay in AIDS progression. The main genetic conditions are described.

\section{Polymorphisms in Chemokine Receptors}

The most important genetic factor related to natural resistance to HIV-1 infection is the presence of the $\Delta 32$ mutation in the CCR5 gene. This mutation occurs in approximately $10 \%$ of Caucasian individuals and represents a deletion of $32 \mathrm{bp}$ resulting in the synthesis of a truncated protein with only 4 of the 7 transmembrane domains required for protein expression at the cell surface [10]. Homozygote individuals for this mutation $(\Delta 32 / \Delta 32)$ are highly resistant to $\mathrm{HIV}-1$ infection [10], mainly to R5 strains, whereas the heterozygotes are susceptible to infection, but exhibit slow progression to AIDS [10]. Nevertheless, this mutation only explains $3.6 \%$ of ESNs [11], suggesting that other mechanisms are involved in natural resistance to infection.

Different polymorphisms in the CCR5 promoter region have been reported and associated with different rates of AIDS progression and natural resistance to HIV infection [12]. Many mutations have been described in this gene, which can produce amino acid substitutions and alter the function of the coreceptor [13]. The polymorphism -2459 $(\mathrm{A} / \mathrm{G})$ in the CCR5 promoter has been associated with delayed progression to AIDS, and seropositive individuals with the $\mathrm{G} / \mathrm{G}$ genotype display slower progression to AIDS 
Table 1. Protective Factors Associated to Different Routes of HIV-1 Transmission

\begin{tabular}{|c|c|c|}
\hline Delta 32 Mutation & Genetic & Sexual and parenteral transmission of R5 strains \\
\hline SNPs in RANTES, MIP- $1 \alpha / \beta$ and $D E F B 1$ genes & Genetic & Sexual and vertical \\
\hline HLA alleles & Genetic & Vertical and sexual \\
\hline Apoptosis of monocytes & Innate immune response & Sexual \\
\hline Production of cytokines by NK cells & Innate immune response & Sexual and parenteral \\
\hline Activity and phenotype of DC & Innate immune response & Vertical \\
\hline $\begin{array}{l}\text { Soluble proteins: APOBEC3G, CAF, defensins, IFNs, LIF, MIP- } \\
1 \alpha / \beta \text {, RANTES, RNases, SDF-1, SLPI and TRIM-5 } \alpha\end{array}$ & Innate immune response & Sexual and vertical \\
\hline
\end{tabular}

than those with the A/A genotype [14]. The CCR5- $\triangle 32$ allele together with the CCR5 -2459G SNP have been associated with a negative effect on the expression of CCR5 in both Thelper cells and monocytes from ESNs, indicating the relationship of these genotypes with natural resistance to HIV infection [12].

Previously, Mummidi et al. reported high genetic variation in the sequence of the CCR5 regulatory region in humans and these CCR5 haplotypes were classified in seven evolutionarily distinct human haplogroups $(\mathrm{HH})$ : $\mathrm{HH}-\mathrm{A},-\mathrm{B}$, $-\mathrm{C},-\mathrm{D},-\mathrm{E},-\mathrm{F}$, and $-\mathrm{G}$; thus, each haplogroup included the combination of a unique SNP in the CCR5 regulatory region, and the presence of either the CCR2 I64V SNP or CCR5$\triangle 32$ mutation [15]. The HH-G2 which includes the CCR5$\Delta 32$ mutation was reported to be protective in children exposed to HIV during prgnancy [16]. The HH-A and HHF2 haplotypes are significantly more frequent in African Americans than in Caucasians, and their presence is associated with disease retardation in SP individuals; in addition, the genotype $\mathrm{HH}-\mathrm{C} / \mathrm{HH}-\mathrm{G} 2$ is related with the strongest protective effects in the same population [17]. Recently, it was observed that the frequency of the HHC/HH-G1 and HH-C/HH-F2 genotypes is higher in ESNs compared to SPs, indicating that these haplogroups could be associated to HIV resistance (J. Vega, unpublished observations, 2009).

In HIV-1-exposed but seronegative intravascular drug users (IDUs) of southeast Asia but not in HIV-1 infected individuals, a guanine to adenine substitution in the 316 position of the CCR5 gene (CCR5 G316A) has been reported; it affects the cellular expression of the coreceptor, suggesting its association with natural resistance to infection [18].

In vitro studies have demonstrated that HIV-1 can use alternative coreceptors, which can exhibit allelic variations that have been associated with resistance to infection and delay in AIDS progression. Among the polymorphisms described in these coreceptors, a genetic variant in the coding region of the CCR2 (CCR2-V64I) produces a change from valine to isoleucine in position 64 of the protein. Some studies have suggested that the CCR2-V64I polymorphism induces negative expression of the CCR5 and CXCR4 molecules at the cell surface, decreasing the availability of susceptible cells to HIV-1 infection [19].

It is known that CCR2 and CCR5 loci are in close proximity, about $10 \mathrm{~kb}$, on chromosome 3, opening the possibility to find non-random association of their alleles. Smith et al., have shown that the mutant allele CCR2-64I is in strong linkage disequilibrium with the CCR5- $\Delta 32$ allele in Afro-Americans, Hispanics and Asian individuals [20]. In addition, several reports indicate that the CCR2-64I allele and the polymorphism CCR5-59653T in the CCR5 promoter are in linkage disequilibrium [21, 22]. The demonstration that these mutant alleles are protective against HIV-1 infection and AIDS progression has important implications for therapy, since these receptors are required for viral entry.

\section{Polymorphisms in Soluble Factors}

The macrophage inflammatory protein-1 alpha (MIP$1 \alpha /$ CCL3) and beta (MIP-1 $\beta /$ CCL4) and the regulated on activation, normal T-cell expressed and secreted (RANTES/CCL5) are CCR5 ligands; several studies have demonstrated the antiviral activity of these proteins [4]. The MIP- $1 \alpha$ and MIP- $1 \beta$ monokines have two genetic variants, $C C L 3 L 1$ and CCL4L1, respectively; they encode proteins that also bind to CCR5, increasing ligand availability, competing with the virus for the coreceptor and inhibiting HIV-1 infection [4]. These genetic variants decrease the probability of acquiring HIV-1 infection and promote recovery of the CD4+ cells after initiation of Highly active antiretroviral treatment (HAART), particularly in individuals with high copy numbers of these alleles, suggesting that lower availability of CCR5 on the cell surface could reduce HIV binding, affecting HIV infection [4]. Recently, a C459T SNP in the intron 1 of MIP-1 $\alpha$ was described whose frequency was higher in ESNs compared to SPs, suggesting that it could increase the efficiency of mRNA processing, thus increasing protein expression (J. Vega, unpublished observations, 2009).

Some polymorphisms in RANTES are associated to resistance. Liu et al. reported that $-28 \mathrm{G}$ and $-403 \mathrm{G} / \mathrm{A}$ SNPs in the upstream non-coding region are associated with increased transcription of the RANTES gene, leading to 
reduced CD4+ $\mathrm{T}$ lymphocyte depletion in HAART naive HIV-1-infected individuals [23].

Polymorphisms in human beta defensins (HBD) have been associated with resistance to HIV-1 infection. We reported that the homozygosity for the A692G polymorphism in DEFBI was significantly more frequent in ESNs than in SPs [24].

\section{Major Histocompatibility Complex (MHC) Alleles}

Alleles of the MHC involved in slow progression to AIDS and resistance to HIV infection have been reported. HLA-B57-restricted CTLs target several gag epitopes that induce much better HIV immune responses through cytolytic destruction of infected cells. HLA-B27 has been associated with delayed HIV-1 escape due to broad reactivity against gag epitopes [25]. Both alleles occur at a higher frequency in LTNPs compared to healthy controls (HCs), suggesting that these molecules have an important antiviral role.

The role of the HLA in HIV-1 vertical transmission had been also explored. An increasing class I HLA concordance between mother/neonate was associated to a higher risk for HIV-1 transmission, that was gene-dose related, suggesting that mother-child MHC discordance could be a protective factor through-out the allogenic immune response. As will be discussed later, and consistent with this hypothesis, we observed that mother/neonate HLA discordance is associated with high mRNA expression of RNase 1 and with the production of an alloantigen-stimulated factor (ASF), a soluble protein with strong anti-HIV-1 activity [26].

\section{IMMUNE RESPONSE DURING HIV-1 EXPOSURE}

\section{Innate Response}

Several investigations have centered on the characteristics of the innate immune response in ESNs, considering that innate immunity is very active on mucosal surfaces that are primary sites of HIV-1 entry.

Apoptosis has been reported not only as a pathogenic mechanism, but also as a mechanism of resistance and control of a variety of infections. We recently determined the role of apoptosis in natural resistance to HIV infection. We found that peripheral blood monocytes in the ESNs group had a greater predisposition to undergo spontaneous apoptosis as well as apoptosis induced by HIV infection in vitro, compared to monocytes from HCs at low risk of HIV infection. This suggests that monocytes could play an important role in the control of HIV infection by undergoing apoptosis [5].

During HIV-1 infection the function and number of NK cells is altered; however, NK cells secrete soluble factors that inhibit HIV infection in vitro. We reported that the expression of IFN- $\gamma$ by phorbol-12-myristate-13-acetate (PMA)/Ionomycin-activated CD56 $6^{\text {bright }}$ NK cells and $\mathrm{CD} 3+/ \mathrm{CD} 56+$ cells was significantly higher in ESNs compared to HCs. Since anti-viral effects of IFN- $\gamma$ are well established, our results suggest that IFN- $\gamma$ production by innate immune cells might be one of the multiple factors involved in controlling the establishment of sexually transmitted HIV-1 infection [6]. Scott-Algara et al. evaluated the lytic activity of NK cells in IDUs, who remained HIV-1 uninfected despite high-risk exposure: there was a higher lytic activity in ESNs than in controls or seroconverters. In addition, NK cells producing IFN- $\gamma$, Tumor necrosis factoralpha (TNF- $\alpha$ ) and the chemokines CCL3, CCL4, and CCL5 were also higher among ESNs [27]. Boulet et al. demonstrated an increased proportion of KIR (Killer immunoglobulin-like receptor)-3DS1 homozygotes in ESNs compared to HIV-1 infected individuals. This finding was associated to an increase in NK cell activation, induction of a strong anti-HIV-1 response and inhibition of the establishment of infection [28], supporting the anti-HIV-1 activity of NK cells.

Dendritic cells (DCs) play a critical role by providing the signals required to induce immune responses. We therefore evaluated the number, phenotype and function of DCs during intrauterine exposure to HIV-1 in uninfected babies born to HIV-1 positive mothers. Intrauterine exposure of the DCs to HIV-1 induces qualitative and quantitative changes in these cells, such as positive regulation of CD80 and CD86 in response to several stimuli, suggesting that these cells could be involved in increasing $T$ lymphocyte activation in these children and could be responsible for the protective immune response observed [7].

\section{Innate Soluble Factors with anti-HIV-1 Activity}

A significant number of soluble factors produced by several cell types have been reported with the capacity to inhibit HIV-1 replication at different steps of the virus life cycle, without eliminating infected cells and through enhancement of specific anti-HIV-1 cellular mechanisms [9]. Among the soluble factors described, those with high levels of anti-HIV-1 activity are the $\beta$-chemokines (RANTES, MIP- $1 \alpha$ and MIP- $\beta$ ) [29], $\alpha$-chemokines such as stromal cellderived factor-1 (SDF-1) [30], the CD8+ cell antiviral factor (CAF) [31], IFN, ribonucleases (RNases) associated with human chorionic gonadotropin [32], ASF [26], the secretory leukocyte protease inhibitor (SLPI) [33], the recently studied apolipoprotein B mRNA-editing enzyme-catalytic polypeptide-like 3G (APOBEC3G) [34], and the $\alpha$ and $\beta$ human defensins $[35,36]$.

\section{Chemokines}

The RANTES (CCL5), MIP-1 $\alpha$ (CCL3), and MIP-1 $\beta$ (CCL4) chemokines are produced by macrophages, NK cells, $\gamma \delta \mathrm{T}$ lymphocyte and T CD8+ lymphocytes [9]. The antiviral role of these chemokines is exerted by competition with the virus for cell adhesion, because they are natural CCR5 ligands, and their affinity also induces coreceptor internalization in early endosomes [29]. The levels of RANTES and MIP- $1 \beta$ are elevated in cultures of HIV-1 envspecific CD4+ T cells from ESNs compared to $\mathrm{HCs}$; in addition, these chemokines were capable of inhibiting replication of macrophage-tropic HIV-1 strains [29]. Additionally, Paxton et al. reported that CD4+ T cells of ESNs are less susceptible to infection and this was associated with the activity of RANTES, MIP- $1 \alpha$, and MIP$1 \beta$ in these cells [37].

The $\alpha$-chemokine SDF-1 is the natural ligand for CXCR4, which is the X4 HIV-1 strain coreceptor. This factor is secreted by bone marrow stromal cells, epithelial cells, monocytes and naïve $\mathrm{T}$ lymphocytes [9]. Studies performed in vitro have demonstrated that SDF-1 binds CXCR4 and down-regulates its expression on cell 
membranes by inducing internalization and lysosomal degradation, decreasing cell susceptibility to infection by $\mathrm{X} 4$ HIV-1 strains [30].

\section{Interferons}

Type I IFN inhibits HIV-1 cell adhesion and induces expression of anti-HIV-1 proteins, such as RNase-L. Moreover, IFN- $\alpha$ induces apoptosis of HIV-1-infected CD4+ $\mathrm{T}$ lymphocytes and inhibits viral replication, diminishing the production of infectious viral particles. In addition, a higher expression of IFN- $\alpha$ was reported in uninfected commercial sex workers with high risk of HIV infection compared to controls, supporting the role of this cytokine in the protection of sexual HIV-1 transmission [38].

ESN NK cells produce greater amounts of IFN- $\gamma$ compared to cells from HCs and the mRNA levels of this cytokine are higher in PBMCs and genital mucosa biopsies of ESNs compared to $\mathrm{HCs}$, suggesting its association to resistance considering that IFN- $\gamma$ inhibits HIV-1 transcription [6]. In addition, Kuhn et al. reported that in sanguineous umbilical cord cells of uninfected children born to HIV-1 positive mothers, the IFN- $\gamma$ concentration is increased, suggesting that this cytokine plays an important role in protection from vertical transmission of HIV [39].

\section{Ribonucleases}

RNases are regulatory enzymes that control several cell processes from alternative RNA splicing to organogenesis, and their therapeutic potential has been suggested and investigated in infectious diseases and cancer. We previously reported that the functional activity of ASF is resistant to high temperature $\left(>90^{\circ} \mathrm{C}\right)$, decreased by RNase inhibitors and inhibited by an antibody against eosinophil-derived neurotoxin (EDN), suggesting that the majority of the antiHIV-1 activity of ASF is due to EDN or a closely related RNase [32]. We recently reported that recombinant RNase A, EDN and angiogenin inhibit primary HIV-1 infection of T cell blasts, suggesting that naturally-occurring RNases play a role in host resistance to HIV-1 and other viral infections [40]. Additionally, we evaluated if maternal-fetal alloantigen stimulation is associated with ASF production and/or placental mRNA expression of three RNases: EDN, RNase 1 and angiogenin. We found that mother/neonate HLA discordance at the $\mathrm{A}$ and $\mathrm{B}$ loci associates with mRNA expression of placental RNases, particularly RNase 1, and with the production of ASF in vitro; these results are consistent with the hypothesis that maternal-fetal HLA differences are important in preventing vertical transmission of certain infectious pathogens, including HIV-1 [26].

\section{Defensins}

Defensins are cysteine-rich cationic peptides with antimicrobial activity against a broad spectrum of microorganisms; they are divided into three groups based on their structure: $\alpha, \beta$ and $\theta$ (the latter only found in nonhuman primates) [35]. Human beta defensins (HBD) are found primarily in mucosa, the main ports of HIV entry. In humans, six HBDs have been reported; HBD-1 is constitutively expressed, whereas HBD-2 and -3 are induced by viral or bacterial products and by cytokines such as IL-1$\beta$ and TNF- $\alpha$ [41]. HBD-2 and -3 mRNAs are induced by HIV-1 in human oral epithelial cells, without infecting the cells. HBD-2 and -3 , but not HBD-1, exhibit strong dose dependent anti-HIV-1 activity, mainly on X4 strains; the inhibition occurs through binding to virions with permanent effect on viral infectivity and downregulation of the HIV-1 coreceptor CXCR4 in PBMCs and T lymphocytes [42]. In addition, Sun et al. showed that both HBD-2 and -3 inhibit infections by R5 and X4 HIV-1 strains in PBMCs. This study also demonstrated that HBD-2 had a direct effect on viral particles in a dose-dependent manner at doses similar to those found in the oral cavity $(100 \mu \mathrm{g} / \mathrm{ml})$ and these defensins inhibited accumulation of early products of reverse transcription [35].

Recently, we observed an increased number of copies of HBD-1, -2 and -3 mRNA in vaginal and endocervical mucosa from ESNs compared to $\mathrm{HCs}$, although these differences were not statistically significant. In addition, a significantly higher expression of HBD-2 and -3 mRNAs was observed in oral mucosa from ESNs compared to the levels in HCs, supporting the role of HBDs during oral exposure to HIV-1 [24]. Thus, the increased HBD expression exhibited by ESNs compared to HCs might reflect a genetic trait associated with resistance to HIV-1 infection.

So far, six human $\alpha$-defensins have been described (HD-1 to -6), playing an important role in innate immunity. HNP-1 to -4 are abundant in polymorphonuclear leukocytes [43], while HD-5 and -6 are produced predominantly by intestinal Paneth cells. HNP-1 to -3 inhibit replication of R5 and X4 HIV-1 strains, including several primary isolates [36]. Chang et al. showed that recombinant HNP-1 inhibited HIV-1 infection in the absence of serum by direct effect on the viral particle. In contrast, in presence of serum the effect is on the cell, affecting steps prior to transcription and nuclear import; likewise, interference with protein kinase $\mathrm{C}(\mathrm{PKC})$ signaling in primary CD4+ T cells by HNP-1 appears to be associated with its inhibitory effect, considering that PKC upregulates HIV transcription through NF- $\mathrm{BB}$ activation and MAPK signaling pathways [44].

It has been reported that $\mathrm{CD} 8+\mathrm{T}$ cells and cervicalvaginal mononuclear cells from ESNs produced 10-fold higher amounts of HNP compared to HCs, and the levels of HNP mRNA and HNP-expressing cells were increased in cervical biopsies of ESNs compared to HCs, suggesting the role of these components in the protective immune response that characterizes ESNs [45]. Supporting their role in natural resistance to HIV infection, Kuhn et al., reported increased levels of HNP-1 to -3 in maternal milk from HIV-1 positive mothers who did not transmit the virus to their infants, compared to transmitting women, suggesting that these proteins are important in inhibiting viral postnatal transmission, probably inactivating viral particles [46]. In addition, immature monocyte-derived DCs from elite and viremic controllers produced higher amounts of HNP-1 to -3 compared to $\mathrm{HCs}$; thus high production of HD-1 to -3 by immature DCs appears to be also a host factor limiting HIV1 replication [47].

\section{Other Soluble Factors}

$\boldsymbol{C A F}$ : It is a soluble factor produced by CD8+ T cells with non-cytotoxic anti-HIV activity by blocking viral transcription [31]. To date, no CD8 T cell-secreted cytokine, chemokine, or inflammatory molecule has been shown to 
identify with the hallmarks that defines the suppressing HIV1 activity of CAF [48]. Stranford et al. reported that CD8+ T cells inhibit HIV replication in acutely infected CD4+ T cells of ESNs and this response efficiently inhibited HIV infection in PBMCs, suggesting that antiviral soluble factors with noncytotoxic activity could be contributing to HIV resistance in this population [49]. In addition, Mackewitz et al., reported that HIV-1 asymptomatic individuals exhibited the highest CAF activity compared to symptomatic and AIDS patients suggesting that CAF could be influencing AIDS progression [50].

SLPI: it is a soluble component of saliva, which is secreted primarily by epithelial cells lining mucosal surfaces and skin, neutrophils, and by lipopolysaccharide-stimulated macrophages [9]. Higher salivary SLPI levels were associated with a decreased risk of HIV-1 infection among infants who were exposed to HIV-1 via breast-feeding after the first month [51]. In agreement with this, in a study carried out by Pillay et al., the SLPI levels in vaginal fluid samples of HIV-1 positive women whose babies became infected were lower than in samples from non-transmitting women [52], implying that SLPI could protect against vertical transmission of HIV-1.

TRIM5 $\alpha$ : Tripartite motif (TRIM) proteins constitute a family of proteins that share a conserved tripartite architecture [53]. TRIM5 $\alpha$ is the only member of these intracellular factors involved in the inhibition of HIV-1 replication. The rhesus monkey TRIM5 $\alpha$ (TRIM5arh) recognizes the incoming HIV-1 core and promotes its premature disassembly or degradation before reverse transcription [54]. Human TRIM5 $\alpha$ has a weak capsidbinding capability compared to TRIM5arh, exhibiting reduced anti-HIV activity; however, it has been postulated that a single amino acid substitution could increase its ability to inhibit HIV-1 replication [55]. Therefore, polymorphisms within TRIM5a might affect susceptibility to HIV-1 infection. Javanbakht et al., reported a higher frequency of the allele TRIM5 $\alpha$ 136Q in ESN compared to HIV-1 infected individuals, suggesting a possible protective role of this variant. In fact, TRIM5 $\alpha 136 \mathrm{Q}$ protein showed higher anti-HIV-1 activity in vitro than TRIM5 $\alpha$ R136 protein supporting its protective effect [56].

Trappin-2: This factor is a protease inhibitor produced at mucosal surfaces by epithelial cells and possibly macrophages, in secreted and active forms known as elafin. Elafin is an antimicrobial and antiinflammatory molecule, which inhibits neutrophil elastase and proteinase 3 [57]. Gosh et al., demonstrated that recombinant Trappin-2/Elafin inhibits both X4/T-tropic IIIB and R5/M-tropic BaL HIV-1 in a dose-dependent manner [58]. In addition, Iqbal et al., using proteomics approach identified high levels of elafin in genital secretions from HIV-resistant women, suggesting its protective role during HIV-1 exposure [59].

APOBEC3G: this enzyme is a protein with cytidine deaminase activity that limits HIV-1 replication. The mechanism involved in this activity is excessive deamination of cytidine to uridine in minus strand reverse transcripts, a process resulting in guanosine to adenosine hypermutation of plus strand DNAs and in the impairment of proviral integration into the host genome [34]. In ESNs, an increasing level of APOBEC3G mRNA in PBMCs was observed compared to HCs; interestingly, the mRNA levels of APOBEC $3 \mathrm{G}$ decreased a year after termination of exposure. These findings were associated with low susceptibility to HIV-1 infection in vitro [60].

\section{Humoral Response}

The humoral response is one of the most important mechanisms in HIV-1 resistance. Indeed, in some ESN individuals, resistance to infection has been associated to HIV-1 specific immunoglobulin A (IgA) production in cervicovaginal secretions, saliva, serum, maternal milk and urethral swabs [2]. Although the mechanisms involved in the production of these antibodies with high specificity and neutralizing capacity is not yet clearly defined, it has been postulated that during HIV-1 exposure some viral particles are processed and presented to $T$ cells, enhancing specific humoral responses [2]. Clerici et al. demonstrated that ESNs IgA binds to a single epitope in the extracellular portion of gp41 (amino acidic residues 581-599: QARILAV). This epitope is involved in the gp41 - gp120 interaction and becomes accessible to specific IgA only after gp120 has bound the CD4 molecule in the target cell; most likely once the antibody binds gp41 the fusion of membranes is blocked. In contrast, IgA from SPs recognizes different epitopes in the gp120 and gp41 transmembrane region, suggesting that recognition of the QARILAV residues is important for resistance to HIV infection, and confirms that qualitative rather than quantitative differences between IgAs produced by ESNs and SPs are more relevant [61]. Supporting the protective role of humoral responses in HIV infection, Hirbord et al., demonstrated an independent association between reduced HIV acquisition and the prior detection of both genital HIV-neutralizing IgA and HIV-specific cellular proliferation [62].

Additionally, higher levels of specific HIV-1 gp160 IgA were found in saliva of HIV-1 negative children, born to HIV-1 positive mothers, who received maternal breastfeeding, compared to $\mathrm{HCs}$, suggesting that a specific IgA response in oral mucosa is important for inhibiting vertical transmission [63]. Considering that oral sex could be a risk factor for HIV-1 infection, Hasselrot et al. evaluated the IgA production in saliva of uninfected men that have oral sex without protection with SP individuals, and reported that this exposure is sufficient to induce IgA1-mediated HIVneutralizing response that can reduce the risk of infection [64]. CCL28 (also called mucosae-associated epithelial chemokine) is a recently described $\mathrm{CC}$ chemokine that binds CCR10 as well as CCR3 receptors, which are expressed by IgA-expressing plasma blasts and plasma cells [65]. CCL28 is detected in trachea, colon, rectum, and exocrine glands such as salivary and mammary glands [66]. Thus, CCL28 is implicated in recruitment of these cells to the mucosal effectors sites. Castelletti et al., evaluated the CCL28 circuit in the context of HIV-1 infection, finding that CCL28 was augmented in breast milk, plasma and saliva of SP and ESN women. Likewise, they showed that CCR3+ and CCR10+ B lymphocytes were increased in these individuals. This suggests a role of CCL28 in the modulation of mucosal immunity in HIV exposure and infection [67].

Despite of all the evidence supporting the role of humoral responses, particularly of IgA in blocking HIV-1 infection, 
several reports, using various cohorts of ESN failed to show this association [68, 69]. In fact, Alexander et al., discussed the possibility that issues such as differences among the ESN cohorts evaluated, variation in processing samples, differences in the prevalence and frequency of unprotected HIV exposure and difficulties to obtain accurate measurements of specific antibody levels, among others, are responsible for the contradictory results. The recommendations to avoid bias in the investigations about $\operatorname{IgA}$ include the use of proper control groups and proper sample preparation in order to ensure that the intrinsic properties of the samples do not influence the results [70].

\section{Cellular Response}

Quantitative and qualitative differences in several effector mechanisms have been described in ESNs, suggesting that some of these parameters might be influenced by virus exposure and could be associated to resistance exhibited by these individuals.

HIV-1 specific CD4+ T cells and PBMCs from ESNs produce high levels of RANTES and MIP-1 $\beta$ [29] and mainly a Th1 cytokine pattern in response to viral peptides, compared to SP individuals [71]. Additionally, Biasin et al. reported significantly higher levels of mRNA for IL-6, IFN$\gamma$, and TNF- $\alpha$ and $\beta$ in cervical samples and PBMCs of ESNs compared to HCs [72]. Taken together, these results suggest that a Th1 response characterized by the high production of IL-2 and IFN- $\gamma$, and low levels of IL-10 could protect against HIV-1 infection.

Immune activation is essential to maintain effective immune responses against pathogens including HIV [72]; however, activation of infected cells induces viral replication. The involvement of immune activation in both, during a protective immune response against HIV and in promoting viral pathogenesis has been suggested by several studies in ESNs. In a Central African cohort, the number of HLA-DR + CD4 $+\mathrm{T}$ cells were found lower in ESNs than in $\mathrm{HCs}$; in addition, in vitro infection of unstimulated PBMC was decreased in ESNs compared to $\mathrm{HCs}$, indicating that low activation and susceptibility may contribute to HIV-1 resistance [73].

In contrast, most reports suggest that ESNs exhibit an immune activation profile. Biasin et al., reported that PBMC from ESNs express higher levels of IL-6, IL-10, IL-12, IFN$\gamma$ and TNF- $\alpha$ and $-\beta$ mRNA; likewise CCR5 and CXCR4 mRNA in cervical mucosa biopsy specimens and HIVspecific IFN- $\gamma$-secreting CD8 $+\mathrm{T}$ lymphocytes in cervical vaginal washes were augmented in ESNs compared to HCs. In addition, they found that CD4+CD25+ and CD8+CD38+ $\mathrm{T}$ lymphocytes were significantly augmented in ESNs, compared to HCs [72]. Interestingly, the level of memory and activation was directly correlated to the viral load of their HIV-1-infected partners, whereas the level of naive and CD28+ $\mathrm{T}$ cells was inversely correlated [74]. All together, these data indicate that immune activation characterizes ESN individuals, suggesting that immunophenotypic profiles were a consequence of HIV-1 exposure or antigenic level.

Several reports indicate that cytotoxic $\mathrm{T}$ lymphocytes (CTL) play an important role in preventing HIV infection. The first demonstration of HIV-1-specific CD8+ lymphocyte responses in the cervix of ESNs was showed by Kaul et al; they demonstrated that CD8+ lymphocytes produced IFN- $\gamma$ in response to HIV-1 [75]. As previously mentioned, the immune activation phenotype and the ability to produce IFN$\gamma$-appears to be important characteristics of CTLs from ESNs [72, 74], suggesting that exposure to HIV-1 influences the maturation profile of subsets of $\mathrm{T}$ lymphocytes that could be associated to HIV resistance.

In addition to the cytotoxic activity of CTLs, CD8+ T cells from ESNs have also shown strong noncytotoxic antiHIV responses, mediated by other soluble factors different than $\beta$-chemokines [49]. Additionally, Pallikkuth et al. reported higher intracellular levels of perforin and granzyme $\mathrm{B}$ expression in $\mathrm{CD} 8+\mathrm{T}$ cells after stimulation with gag p24 antigen in ESNs compared to HCs [76].

\section{CONCLUSIONS}

CCR5 $\Delta 32$ polymorphism remains the main genetic factor that confers resistance to HIV-1. However, phenotypic and functional characteristics of immune cells and the antiviral activity of soluble factors have become the aim of several studies trying to determine their association with protection against HIV infection. As described in this review, although some of these cells and molecules have been clearly associated with the phenomenon of natural resistance to HIV-1 infection, the mechanisms responsible for viral inhibition are not fully understood. A better understanding of this phenomenon is essential for the development of effective vaccines to prevent viral infection. In this sense, the groups of ESNs, ellite controllers and LTNPs will continue to be the main target populations to address the variety of mechanisms which allow controlling HIV infection.

\section{ACKNOWLEDGEMENTS}

This work was supported by The Committee for Research Development, "sostenibilidad 2009-2011" from the University of Antioquia and COLCIENCIAS (grant 111534319143). We thank Anne-Lise Haenni for all her constructive comments.

\section{REFERENCES}

[1] UNAIDS. AIDS epidemic update. Geneva: UNAIDS/WHO 2009.

[2] Piacentini L, Fenizia C, Naddeo V, Clerici M. Not just sheer luck! Immune correlates of protection against HIV-1 infection. Vaccine 2008; 26(24): 3002-7.

[3] Walker BD. Elite control of HIV Infection: implications for vaccines and treatment. Top HIV Med 2007;15(4): 134-6.

[4] Modi WS, Lautenberger J, An P, et al. Genetic variation in the CCL18-CCL3-CCL4 chemokine gene cluster influences HIV Type 1 transmission and AIDS disease progression. Am J Hum Genet 2006; 79(1): 120-8.

[5] Velilla PA, Hoyos A, Rojas M, Patino PJ, Velez LA, Rugeles MT. Apoptosis as a mechanism of natural resistance to HIV-1 infection in an exposed but uninfected population. J Clin Virol 2005; 32(4): 329-35.

[6] Montoya CJ, Velilla PA, Chougnet C, Landay AL, Rugeles MT. Increased IFN-gamma production by NK and CD3+/CD56+ cells in sexually HIV-1-exposed but uninfected individuals. Clin Immunol 2006; 120(2): 138-46.

[7] Velilla PA, Montoya CJ, Hoyos A, Moreno ME, Chougnet C, Rugeles MT. Effect of intrauterine HIV-1 exposure on the frequency and function of uninfected newborns' dendritic cells. Clin Immunol 2008; 126(3): 243-50.

[8] Kaul R, Dong T, Plummer FA, et al. CD8(+) lymphocytes respond to different HIV epitopes in seronegative and infected subjects. J Clin Invest 2001 May;107(10):1303-10. 
[9] Zapata W, Montoya CJ, Rugeles MT. Soluble factors with inhibitory activity against type 1 Human Immunodeficiency Virus. Biomedica 2006; 26(3): 451-66

[10] Galvani AP, Novembre J. The evolutionary history of the CCR5Delta32 HIV-resistance mutation. Microbes Infect 2005; 7(2): 3029 .

[11] Diaz FJ, Vega JA, Patino PJ, et al. Frequency of CCR5 delta-32 mutation in human immunodeficiency virus (HIV)-seropositive and HIV-exposed seronegative individuals and in general population of Medellin, Colombia. Mem Inst Oswaldo Cruz 2000; 95(2): 237-42.

[12] Hladik F, Liu H, Speelmon E, et al. Combined effect of CCR5Delta32 heterozygosity and the CCR5 promoter polymorphism $2459 \mathrm{~A} / \mathrm{G}$ on CCR5 expression and resistance to human immunodeficiency virus type 1 transmission. J Virol 2005; 79(18): 11677-84.

[13] Blanpain C, Lee B, Tackoen M, et al. Multiple nonfunctional alleles of CCR5 are frequent in various human populations. Blood 2000; 96(5): 1638-45.

[14] Knudsen TB, Kristiansen TB, Katzenstein TL, Eugen-Olsen J. Adverse effect of the CCR5 promoter -2459A allele on HIV-1 disease progression. J Med Virol 2001; 65(3): 441-4.

[15] Mummidi S, Bamshad M, Ahuja SS, et al. Evolution of human and non-human primate $\mathrm{CC}$ chemokine receptor 5 gene and mRNA. Potential roles for haplotype and mRNA diversity, differential haplotype-specific transcriptional activity, and altered transcription factor binding to polymorphic nucleotides in the pathogenesis of HIV-1 and simian immunodeficiency virus. J Biol Chem 2000; 275(25): 18946-61.

[16] Ometto L, Zanchetta M, Mainardi M, et al. Co-receptor usage of HIV-1 primary isolates, viral burden, and CCR5 genotype in mother-to-child HIV-1 transmission. Aids 2000; 14(12): 1721-9.

[17] Gonzalez E, Bamshad M, Sato N, et al. Race-specific HIV-1 disease-modifying effects associated with CCR5 haplotypes. Proc Natl Acad Sci U S A 1999; 96(21): 12004-9.

[18] Capoulade-Metay C, Ma L, Truong LX, et al. New CCR5 variants associated with reduced HIV coreceptor function in South East Asia. Aids 2004; 18(17): 2243-52.

[19] Sabbe R, Picchio GR, Pastore C, et al. Donor- and liganddependent differences in $\mathrm{C}-\mathrm{C}$ chemokine receptor 5 reexpression. J Virol 2001; 75(2): 661-71

[20] Smith MW, Dean M, Carrington M, et al. Contrasting genetic influence of CCR2 and CCR5 variants on HIV-1 infection and disease progression. Hemophilia Growth and Development Study (HGDS), Multicenter AIDS Cohort Study (MACS), Multicenter Hemophilia Cohort Study (MHCS), San Francisco City Cohort (SFCC), ALIVE Study. Science 1997; 277(5328): 959-65.

[21] Lewandowska M, Jagodzinski PP, Trzeciak WH. Decreased density of the CCR5 receptor on the surface of CD4+ lymphocytes and monocytes/macrophages is associated with the CCR5-59653T transition in the promoter region. Folia Histochem Cytobiol 2002; 40(2): 101-2.

[22] Martinson JJ, Hong L, Karanicolas R, Moore JP, Kostrikis LG. Global distribution of the CCR2-64I/CCR5-59653T HIV-1 diseaseprotective haplotype. Aids 2000; 14(5): 483-9.

[23] Liu H, Chao D, Nakayama EE, et al. Polymorphism in RANTES chemokine promoter affects HIV-1 disease progression. Proc Natl Acad Sci U S A 1999; 96(8): 4581-5.

[24] Zapata W, Weber J, Estrada H, et al. Increased levels of human beta-defensins mrna in sexually HIV-1 exposed but uninfected individuals. Curr HIV Res 2008; 6(6): 531-8.

[25] Streeck H, Lichterfeld M, Alter G, et al. Recognition of a defined region within $\mathrm{p} 24$ gag by $\mathrm{CD} 8+\mathrm{T}$ cells during primary human immunodeficiency virus type 1 infection in individuals expressing protective HLA class I alleles. J Virol 2007; 81(14): 7725-31.

[26] Bedoya VI, Jaimes FA, Delgado JC, et al. Fetal-maternal HLA-A and $-\mathrm{B}$ discordance is associated with placental RNase expression and anti-HIV-1 activity. Curr HIV Res 2008; 6(4): 380-7.

[27] Scott-Algara D, Truong LX, Versmisse P, et al. Cutting edge: increased NK cell activity in HIV-1-exposed but uninfected Vietnamese intravascular drug users. J Immunol 2003; 171(11): 5663-7.

[28] Boulet S, Sharafi S, Simic N, et al. Increased proportion of KIR3DS1 homozygotes in HIV-exposed uninfected individuals. Aids 2008; 22(5): 595-9.

[29] Furci L, Scarlatti G, Burastero S, et al. Antigen-driven C-C chemokine-mediated HIV-1 suppression by CD4(+) T cells from exposed uninfected individuals expressing the wild-type CCR-5 allele. J Exp Med 1997; 186(3): 455-60.

[30] Doranz BJ, Grovit-Ferbas K, Sharron MP, et al. A small-molecule inhibitor directed against the chemokine receptor CXCR4 prevents its use as an HIV-1 coreceptor. J Exp Med 1997; 186(8): 1395-400.

[31] Levy JA. The search for the CD8+ cell anti-HIV factor (CAF). Trends Immunol 2003; 24(12): 628-32.

[32] Rugeles MT, Trubey CM, Bedoya VI, et al. Ribonuclease is partly responsible for the HIV-1 inhibitory effect activated by HLA alloantigen recognition. Aids 2003; 17(4): 481-6.

[33] McNeely TB, Shugars DC, Rosendahl M, Tucker C, Eisenberg SP, Wahl SM. Inhibition of human immunodeficiency virus type 1 infectivity by secretory leukocyte protease inhibitor occurs prior to viral reverse transcription. Blood 1997; 90(3): 1141-9.

[34] Newman EN, Holmes RK, Craig HM, et al. Antiviral function of APOBEC3G can be dissociated from cytidine deaminase activity. Curr Biol 2005; 15(2): 166-70.

[35] Sun L, Finnegan CM, Kish-Catalone T, et al. Human betadefensins suppress human immunodeficiency virus infection: potential role in mucosal protection. J Virol 2005; 79(22): 1431829.

[36] Zhang L, Yu W, He T, et al. Contribution of human alpha-defensin 1,2 , and 3 to the anti-HIV-1 activity of CD8 antiviral factor. Science 2002; 298(5595): 995-1000.

[37] Paxton WA, Martin SR, Tse D, et al. Relative resistance to HIV-1 infection of CD4 lymphocytes from persons who remain uninfected despite multiple high-risk sexual exposure. Nat Med 1996; 2(4): 412-7.

[38] Hirbod T, Nilsson J, Andersson S, et al. Upregulation of interferonalpha and RANTES in the cervix of HIV-1-seronegative women with high-risk behavior. J Acquir Immune Defic Syndr 2006; 43(2): 137-43.

[39] Kuhn L, Coutsoudis A, Moodley D, et al. Interferon-gamma and interleukin-10 production among HIV-1-infected and uninfected infants of HIV-1-infected mothers. Pediatr Res 2001; 50(3): 412-6.

[40] Bedoya VI, Boasso A, Hardy AW, Rybak S, Shearer GM, Rugeles MT. Ribonucleases in HIV type 1 inhibition: effect of recombinant RNases on infection of primary $\mathrm{T}$ cells and immune activationinduced RNase gene and protein expression. AIDS Res Hum Retroviruses 2006; 22(9): 897-907.

[41] Lehrer RI, Ganz T. Defensins of vertebrate animals. Curr Opin Immunol 2002; 14(1): 96-102.

[42] Yang D, Biragyn A, Kwak LW, Oppenheim JJ. Mammalian defensins in immunity: more than just microbicidal. Trends Immunol 2002; 23(6): 291-6.

[43] Ganz T. Defensins: antimicrobial peptides of innate immunity. Nat Rev Immunol 2003; 3(9): 710-20.

[44] Chang TL, Vargas J, Jr., DelPortillo A, Klotman ME. Dual role of alpha-defensin-1 in anti-HIV-1 innate immunity. J Clin Invest 2005; 115(3): 765-73.

[45] Trabattoni D, Caputo SL, Maffeis G, et al. Human alpha defensin in HIV-exposed but uninfected individuals. J Acquir Immune Defic Syndr 2004; 35(5): 455-63.

[46] Kuhn L, Trabattoni D, Kankasa C, et al. Alpha-defensins in the prevention of HIV transmission among breastfed infants. J Acquir Immune Defic Syndr 2005; 39(2): 138-42.

[47] Rodriguez-Garcia M, Climent N, Oliva H, et al. Increased alphadefensins 1-3 production by dendritic cells in HIV-infected individuals is associated with slower disease progression. PLoS One 5(2): e9436.

[48] Chang TL, Francois F, Mosoian A, Klotman ME. CAF-mediated human immunodeficiency virus (HIV) type 1 transcriptional inhibition is distinct from alpha-defensin-1 HIV inhibition. J Virol 2003; 77(12): 6777-84.

[49] Stranford SA, Skurnick J, Louria D, et al. Lack of infection in HIV-exposed individuals is associated with a strong CD8(+) cell noncytotoxic anti-HIV response. Proc Natl Acad Sci U S A 1999; 96(3): 1030-5.

[50] Mackewicz CE, Ortega HW, Levy JA. CD8+ cell anti-HIV activity correlates with the clinical state of the infected individual. J Clin Invest 1991; 87(4): 1462-6.

[51] Farquhar C, VanCott TC, Mbori-Ngacha DA, et al. Salivary secretory leukocyte protease inhibitor is associated with reduced transmission of human immunodeficiency virus type 1 through breast milk. J Infect Dis 2002; 186(8): 1173-6. 
[52] Pillay K, Coutsoudis A, Agadzi-Naqvi AK, Kuhn L, Coovadia HM, Janoff EN. Secretory leukocyte protease inhibitor in vaginal fluids and perinatal human immunodeficiency virus type 1 transmission. J Infect Dis 2001; 183(4): 653-6.

[53] Reymond A, Meroni G, Fantozzi A, et al. The tripartite motif family identifies cell compartments. EMBO J 2001; 20(9): 214051 .

[54] Stremlau M, Perron M, Lee M, et al. Specific recognition and accelerated uncoating of retroviral capsids by the TRIM5alpha restriction factor. Proc Natl Acad Sci U S A 2006; 103(14): 5514-9.

[55] Yap MW, Nisole S, Stoye JP. A single amino acid change in the SPRY domain of human Trim5alpha leads to HIV-1 restriction. Curr Biol 2005; 15(1): 73-8.

[56] Javanbakht H, An P, Gold B, et al. Effects of human TRIM5alpha polymorphisms on antiretroviral function and susceptibility to human immunodeficiency virus infection. Virology 2006; 354(1): $15-27$

[57] Moreau T, Baranger K, Dade S, Dallet-Choisy S, Guyot N, Zani ML. Multifaceted roles of human elafin and secretory leukocyte proteinase inhibitor (SLPI), two serine protease inhibitors of the chelonianin family. Biochimie 2008; 90(2): 284-95.

[58] Ghosh M, Shen Z, Fahey JV, Cu-Uvin S, Mayer K, Wira CR. Trappin-2/Elafin: a novel innate anti-human immunodeficiency virus-1 molecule of the human female reproductive tract. Immunology 2010; 129(2): 207-19.

[59] Iqbal SM, Ball TB, Levinson P, et al. Elevated elafin/trappin-2 in the female genital tract is associated with protection against HIV acquisition. Aids 2009; 23(13): 1669-77.

[60] Biasin M, Piacentini L, Lo Caputo S, et al. Apolipoprotein B mRNA-editing enzyme, catalytic polypeptide-like 3G: a possible role in the resistance to HIV of HIV-exposed seronegative individuals. J Infect Dis 2007; 195(7): 960-4.

[61] Clerici M, Barassi C, Devito C, et al. Serum IgA of HIV-exposed uninfected individuals inhibit HIV through recognition of a region within the alpha-helix of gp41. Aids 2002; 16(13): 1731-41.

[62] Hirbod T, Kaul R, Reichard C, et al. HIV-neutralizing immunoglobulin A and HIV-specific proliferation are independently associated with reduced HIV acquisition in Kenyan sex workers. Aids 2008; 22(6): 727-35.

[63] Farquhar C, VanCott $\mathrm{T}$, Bosire R, et al. Salivary human immunodeficiency virus (HIV)-1-specific immunoglobulin A in HIV-1-exposed infants in Kenya. Clin Exp Immunol 2008; 153(1): $37-43$
[64] Hasselrot K, Bratt G, Hirbod T, et al. Orally exposed uninfected individuals have systemic anti-HIV responses associating with partners' viral load. Aids; 24(1): 35-43.

[65] Hieshima K, Kawasaki Y, Hanamoto $\mathrm{H}$, et al. CC chemokine ligands 25 and 28 play essential roles in intestinal extravasation of IgA antibody-secreting cells. J Immunol 2004; 173(6): 3668-75.

[66] Pan J, Kunkel EJ, Gosslar U, et al. A novel chemokine ligand for CCR10 and CCR3 expressed by epithelial cells in mucosal tissues. J Immunol 2000; 165(6): 2943-9.

[67] Castelletti E, Lo Caputo S, Kuhn L, et al. The mucosae-associated epithelial chemokine (MEC/CCL28) modulates immunity in HIV infection. PLoS One 2007; 2(10): e969.

[68] Dorrell L, Hessell AJ, Wang M, et al. Absence of specific mucosal antibody responses in HIV-exposed uninfected sex workers from the Gambia. Aids 2000; 14(9): 1117-22

[69] Fiore JR, Laddago V, Lepera A, et al. Limited secretory-IgA response in cervicovaginal secretions from HIV-1 infected, but not high risk seronegative women: lack of correlation to genital viral shedding. New Microbiol 2000; 23(1): 85-92.

[70] Alexander R, Mestecky J. Neutralizing antibodies in mucosal secretions: IgG or IgA? Curr HIV Res 2007; 5(6): 588-93.

[71] Mazzoli S, Trabattoni D, Lo Caputo S, et al. HIV-specific mucosal and cellular immunity in HIV-seronegative partners of HIVseropositive individuals. Nat Med 1997; 3(11): 1250-7.

[72] Biasin M, Caputo SL, Speciale L, et al. Mucosal and systemic immune activation is present in human immunodeficiency virusexposed seronegative women. J Infect Dis 2000; 182(5): 1365-74

[73] Begaud E, Chartier L, Marechal V, et al. Reduced CD4 T cell activation and in vitro susceptibility to HIV-1 infection in exposed uninfected Central Africans. Retrovirology 2006; 3: 35 .

[74] Suy A, Castro P, Nomdedeu M, et al. Immunological profile of heterosexual highly HIV-exposed uninfected individuals: predominant role of CD4 and CD8 T-cell activation. J Infect Dis 2007; 196(8): 1191-201.

[75] Kaul R, Plummer FA, Kimani J, et al. HIV-1-specific mucosal CD8+ lymphocyte responses in the cervix of HIV-1-resistant prostitutes in Nairobi. J Immunol 2000; 164(3): 1602-11.

[76] Pallikkuth S, Wanchu A, Bhatnagar A, Sachdeva RK, Sharma M. Human immunodeficiency virus (HIV) gag antigen-specific Thelper and granule-dependent CD8 T-cell activities in exposed but uninfected heterosexual partners of HIV type 1-infected individuals in North India. Clin Vaccine Immunol 2007; 14(9): 1196-202.

This is an open access article licensed under the terms of the Creative Commons Attribution Non-Commercial License (http://creativecommons.org/licenses/by-nc/ 3.0/) which permits unrestricted, non-commercial use, distribution and reproduction in any medium, provided the work is properly cited. 